\title{
A simple and efficient automated microvolume radiosynthesis of $\left[{ }^{18} \mathrm{~F}\right]$ Florbetaben
}

Ksenia Lisova ${ }^{1,2,3}$ (D) Jia Wang ${ }^{2,3,4}$, Philip H. Chao ${ }^{2,3,4}$ and R. Michael van Dam 1,2,3**

\author{
*Correspondence: mvandam@ \\ mednet.ucla.edu \\ ${ }^{1}$ Physics \& Biology in Medicine \\ Interdepartmental Graduate \\ Program, University of California Los \\ Angeles, Los Angeles, CA, USA \\ ${ }^{2}$ Crump Institute for Molecular \\ Imaging, University of California Los \\ Angeles, Los Angeles, CA, USA \\ Full list of author information is \\ available at the end of the article
}

\begin{abstract}
Background: Current automated radiosynthesizers are generally optimized for producing large batches of PET tracers. Preclinical imaging studies, however, often require only a small portion of a regular batch, which cannot be economically produced on a conventional synthesizer. Alternative approaches are desired to produce small to moderate batches to reduce cost and the amount of reagents and radioisotope needed to produce PET tracers with high molar activity. In this work we describe the first reported microvolume method for production of $\left[{ }^{18} \mathrm{~F}\right]$ Florbetaben for use in imaging of Alzheimer's disease.
\end{abstract}

Procedures: The microscale synthesis of $\left[{ }^{18} \mathrm{~F}\right]$ Florbetaben was adapted from conventional-scale synthesis methods. Aqueous $\left[{ }^{18} \mathrm{~F}\right]$ fluoride was azeotropically dried with $\mathrm{K}_{2} \mathrm{CO}_{3} / \mathrm{K}_{222}(275 / 383 \mathrm{nmol})$ complex prior to radiofluorination of the Bocprotected precursor $(80 \mathrm{nmol})$ in $10 \mu \mathrm{L}$ DMSO at $130^{\circ} \mathrm{C}$ for $5 \mathrm{~min}$. The resulting intermediate was deprotected with $\mathrm{HCl}$ at $90^{\circ} \mathrm{C}$ for 3 min and recovered from the chip in aqueous acetonitrile solution. The crude product was purified via analytical scale HPLC and the collected fraction reformulated via solid-phase extraction using a miniature C18 cartridge.

Results: Starting with $270 \pm 100 \mathrm{MBq}(n=3)$ of $\left[{ }^{18} \mathrm{~F}\right]$ Fluoride, the method affords formulated product with $49 \pm 3 \%$ (decay-corrected) yield, $>98 \%$ radiochemical purity and a molar activity of $338 \pm 55 \mathrm{GBq} / \mu \mathrm{mol}$. The miniature C18 cartridge enables efficient elution with only $150 \mu \mathrm{L}$ of ethanol which is diluted to a final volume of 1.0 $\mathrm{mL}$, thus providing a sufficient concentration for in vivo imaging. The whole procedure can be completed in 55 min.

Conclusions: This work describes an efficient and reliable procedure to produce $\left[{ }^{18} \mathrm{~F}\right]$ Florbetaben in quantities sufficient for large-scale preclinical applications. This method provides very high yields and molar activities compared to reported literature methods. This method can be applied to higher starting activities with special consideration given to automation and radiolysis prevention.

Keywords: Microfluidics, Radiochemistry, Beta-amyloid imaging, Florbetaben, Molar activity, Droplet synthesis, Automation, Radiolysis 


\section{Background}

Positron emission tomography (PET) is a powerful molecular imaging tool with extensive applications in disease diagnostics and drug development, among other areas. Many tracers (labeled with positron-emitting isotopes) have been developed that can bind to specific molecular targets in vivo and allow tracking of their dynamics and location throughout the whole body. For example, a number of tracers were developed to target amyloid-beta $(\mathrm{A} \beta)$ plaques which are correlated with the progression of Alzheimer's disease (Hardy and Selkoe 2002), and it is believed that the high sensitivity and specificity of PET can aid in early diagnosis of dementia and its grading (Ossenkoppele et al. 2015).

$\mathrm{N}$-methyl- $\left[{ }^{11} \mathrm{C}\right] 2-\left(4^{\prime}\right.$-methylaminophenyl)-6-hydroxybenzothiazole $\quad\left(\left[{ }^{11} \mathrm{C}\right] \mathrm{PIB}\right), \quad$ a radiofluorinated thioflavin $\mathrm{T}$ analogue, was the first selective radiotracer for Alzheimer's disease research and diagnostics (Klunk et al. 2004). However, the short half-life of carbon-11 (20 $\mathrm{min}$ ) restricts the access to this probe, especially at locations not having their own cyclotron and expertise in ${ }^{11} \mathrm{C}$-radiochemistry, thus alternative tracers labeled with longer-lived isotopes (e.g. fluorine-18, $t_{1 / 2}=110 \mathrm{~min}$ ) were developed. In fact, several ${ }^{18}$ F-labeled amyloid imaging agents have been reported, including $\left[{ }^{18} \mathrm{~F}\right]$ Florbetapir, $\left[{ }^{18} \mathrm{~F}\right]$ Florbetaben and $\left[{ }^{18} \mathrm{~F}\right]$ Flutemetamol, and were approved for use in clinical practice in the United States (Yeo et al. 2015). In this work we focus on $\left[{ }^{18} \mathrm{~F}\right]$ Florbetaben (also known by several other names: $\left[{ }^{18} \mathrm{~F}\right] \mathrm{FBB},\left[{ }^{18} \mathrm{~F}\right] \mathrm{BAY} 94-9172$, NeuraCeq, $\left.\left[{ }^{18} \mathrm{~F}\right] \mathrm{AV} 1,\left[{ }^{18} \mathrm{~F}\right] \mathrm{AV} 1 / \mathrm{ZK}\right)$, a stilbene derivative that was designed to selectively bind to $A \beta$ plaques (Zhang et al. 2005). $\left[{ }^{18} \mathrm{~F}\right]$ Florbetaben $\left(\left[{ }^{18} \mathrm{~F}\right] \mathrm{FBB}\right.$ ) and other similar tracers are extremely useful for studying cases of Alzheimer's disease both in the clinic and in research. Clinically, the tracers are used for accurate dementia grading and early detection in at-risk populations. In research, these tracers are useful for testing of new treatments and understanding their influence on disease progression (Brendel et al. 2016; Brendel et al. 2018; Sabri et al. 2015; Blume et al. 2018; Rominger et al. 2013; Sacher et al. 2019). Though the influence of molar activity in amyloid imaging has not been widely reported, in general preclinical imaging in small animal models (e.g. mice) requires tracers with high molar activity (Kung and Kung 2005; Jagoda et al. 2004; Sergeev et al. 2018). There seem to be mixed reports about the importance of molar activity in small animal amyloid imaging (Snellman et al. 2014), but it has been reported that detection of relatively immature (small and diffuse) amyloid lesions in mouse brain (with $\left[{ }^{11} \mathrm{C}\right] \mathrm{PIB}$ ) is especially sensitive to molar activity (Maeda et al. 2007).

Further development and use of these tracers in a preclinical context is hindered by limited access and/or high cost of tracers such as $\left[{ }^{18} \mathrm{~F}\right]$ Florbetaben. Current methods and reagent kits are optimized for large-scale production, making economical production of small batches not possible with current radiosynthesizer technologies. To address these concerns, we sought to optimize production of $\left[{ }^{18} \mathrm{~F}\right]$ Florbetaben at the microliter scale while providing high-quality product suitable for in vivo preclinical applications. Due to the small physical size of microfluidic radiosynthesizers, very low amounts of reagents and radionuclide are needed, reducing the cost of materials and equipment, and required radiation shielding (Dooraghi et al. 2013; Elizarov et al. 2010; Lebedev et al. 2012; Keng et al. 2016; Wang et al. 2017). The synthesis of multiple tracers and prosthetic groups have been successfully implemented in microliter droplet-reactor format, including $\left[{ }^{18} \mathrm{~F}\right] \mathrm{FDG}$ (Wang et al. 2017; Keng et al. 2012), 
$\left[{ }^{18} \mathrm{~F}\right]$ Fallypride (Wang et al. 2017; Javed et al. 2014; Wang et al. 2019a), $\left[{ }^{18} \mathrm{~F}\right] \mathrm{FET}$ (Lisova et al. 2019), $\left[{ }^{18} \mathrm{~F}\right] \mathrm{FDOPA}$ (Wang et al. 2019b), $\left[{ }^{18} \mathrm{~F}\right] \mathrm{AMBF}_{3}$-TATE (Lisova et al. 2018), $\left[{ }^{18} \mathrm{~F}\right] \mathrm{SFB}$ (Kim et al. 2019) and sulfonyl $\left[{ }^{18} \mathrm{~F}\right]$ fluoride (Fiel et al. 2015), providing a strong suggestion that $\left[{ }^{18} \mathrm{~F}\right]$ Florbetaben could also be implemented.

To date there are no reported methods (droplet or other formats) for microfluidic synthesis of $\left[{ }^{18} \mathrm{~F}\right]$ Florbetaben. Synthesis of $\left[{ }^{18} \mathrm{~F}\right]$ Florbetaben via conventional methods typically follows a 2-step synthesis using an N-Boc-protected precursor (Zhang et al. 2005; Rominger et al. 2013; Patt et al. 2010; Wang et al. 2013), followed by semipreparative HPLC purification and reformulation via C18 solid-phase extraction (SPE) (Rominger et al. 2013; Patt et al. 2010; Rowe et al. 2008). Here we develop an automated microliter droplet-based synthesis of this probe and demonstrate the benefits of using small scale production. The synthesis is performed in a microdroplet reactor, with purification via analytical-scale HPLC, and formulation carried out on a system built in-house for small-volume SPE. The low-cost approach presented here uses tiny amounts of reagents and achieves high molar activity without the need for high starting activity (as required in traditional radiosynthesizers), producing high-quality $\left[{ }^{18} \mathrm{~F}\right]$ Florbetaben readily applicable for small animal imaging and with potential of scaling up to clinical doses.

\section{Materials and methods}

\section{Reagents}

No-carrier-added $\left[{ }^{18} \mathrm{~F}\right]$ fluoride was produced by the ${ }^{18} \mathrm{O}(\mathrm{p}, \mathrm{n}){ }^{18} \mathrm{~F}$ reaction from $\left[{ }^{18} \mathrm{O}\right] \mathrm{H}_{2} \mathrm{O}$ (84\% isotopic purity, Zevacor Pharma, Noblesville, IN, USA) in an RDS-112 cyclotron (Siemens; Knoxville, TN, USA) at $11 \mathrm{MeV}$ using a $1 \mathrm{~mL}$ tantalum target with havar foil. Acetonitrile (MeCN; anhydrous, 99.8\%), methanol (MeOH; anhydrous, 99.8\%), ethanol (EtOH; 200 proof, > 99.5\%), hydrochloric acid ( $\mathrm{HCl} ; 1 \mathrm{M})$, dimethylsulfoxide (DMSO, 98\%), deionized (DI) water, and polyethylene glycol 400 (PEG 400) were purchased from Millipore Sigma (St. Louis, MO, USA). All reagents were used as received without further purification. N-Boc protected mesylate FBB precursor ([methanesulfonic acid 2-\{2-[2-(4-\{2-[4-(tert-butoxycarbonyl-methyl-amino)-phenyl]-vinyl\}phenoxy)-ethoxy]-ethoxy\}-ethyl ester) and FBB reference standard (4-[(E)-2-(4-\{2-[2-(2$\left[{ }^{18} \mathrm{~F}\right]$ fluoroethoxy) ethoxy] ethoxy\} phenyl) vinyl $]-\mathrm{N}$-methylaniline) were generously provided by Life Molecular Imaging $\mathrm{GmbH}$ as a part of $\left[{ }^{18} \mathrm{~F}\right]$ Florbetaben synthesis kits. Kryptofix $222\left(\mathrm{~K}_{222}\right)$ and potassium carbonate $\left(\mathrm{K}_{2} \mathrm{CO}_{3}\right)$ were purchased from ABX $\mathrm{GmbH}$ (Radeberg, Germany). Sodium phosphate dibasic $\left(\mathrm{Na}_{2} \mathrm{HPO}_{4} \cdot 7 \mathrm{H}_{2} \mathrm{O}\right)$ and sodium phosphate monobasic $\left(\mathrm{NaH}_{2} \mathrm{PO}_{4} \cdot \mathrm{H}_{2} \mathrm{O}\right)$ were purchased from Fisher Scientific (Thermo Fisher Scientific, Waltham, MA). Ultrapure $18 \mathrm{M} \Omega$ water was acquired through a MilliQ Integral 3 purification system (Millipore Sigma, St. Louis, MO, USA).

Dry scavenger mix (used in multiple steps of the reaction), consisting of sodium ascorbate with L-ascorbic acid $(87: 13 \mathrm{w} / \mathrm{w})$, was obtained from the $\left[{ }^{18} \mathrm{~F}\right]$ Florbetaben production kits provided by Life Molecular Imaging GmbH. HPLC mobile phase was prepared by first dissolving $1.785 \mathrm{~g}$ of $\mathrm{Na}_{2} \mathrm{HPO}_{4} \cdot 7 \mathrm{H}_{2} \mathrm{O}$ and $0.461 \mathrm{~g}$ of $\mathrm{NaH}_{2} \mathrm{PO}_{4} \cdot \mathrm{H}_{2} \mathrm{O}$ in $0.40 \mathrm{~L}$ of $18 \mathrm{M} \Omega \mathrm{H}_{2} \mathrm{O}$ to make $25 \mathrm{mM}$ phosphate buffer (pH 7.2), then adding in $0.60 \mathrm{~L}$ of $\mathrm{MeCN}$. Collection mixture to recover the crude $\left[{ }^{18} \mathrm{~F}\right]$ Florbetaben from the chip consisted of $\mathrm{MeCN}$ mixed with $33 \mathrm{mg} / \mathrm{mL}$ dry scavenger mix in DI water $(1: 1, \mathrm{v} / \mathrm{v})$. 
Stabilization / dilution solution for formulation contained $39 \mathrm{mg} / \mathrm{mL}$ of dry scavenger mix in a 4:13 (v/v) mixture of PEG 400 and DI water.

\section{Analytical methods}

A calibrated ion chamber (CRC 25-PET, Capintec, Florham Park, NJ, USA) was used to perform radioactivity measurements. For radio-thin-layer chromatography (radio-TLC) analysis, reverse phase TLC plates (RP-18 silica gel 60 F254 sheets; aluminum backing; Millipore Sigma, St. Louis, MO, USA) were cut into $15 \times 60 \mathrm{~mm}$ pieces (with $40 \mathrm{~mm}$ developing distance), spotted with $1 \mu \mathrm{L}$ of the sample and developed in $90 \%(\mathrm{v} / \mathrm{v})$ $\mathrm{MeCN}$ in $\mathrm{H}_{2} \mathrm{O}$. TLC plates were analyzed with a Cerenkov luminescence imaging system as previously described (Wang et al. 2020) or a conventional radio-TLC scanner (miniGita star, Raytest, Inc., Wilmington, NC, USA). Retention factors of the observed radioactive species were: 0.0 ( $\left[{ }^{18} \mathrm{~F}\right]$ fluoride), $0.4 \quad\left(\left[{ }^{18} \mathrm{~F}\right] \mathrm{FBB}\right)$, and 0.8 (fluorinated intermediate).

Radio-HPLC analysis (and purification) were performed on an analytical-scale Smartline HPLC system (Knauer, Berlin, Germany) with $200 \mu \mathrm{L}$ injection loop, a pump (Model 1000), degasser (Model 5050), UV detector (Model 2500) and a radiometric detector (Bioscan B-FC-4000, Bioscan Inc., Washington DC, USA). Samples were separated using a $\mathrm{C} 18$ column (Luna, $5 \mu \mathrm{m}$ particles, $100 \AA$ pores, $250 \times 4.6 \mathrm{~mm}$, Phenomenex, Torrance, CA, USA) with guard column (SecurityGuard C18, Phenomenex). UV absorbance was measured at $254 \mathrm{~nm}$. Using isocratic conditions with a MeCN: $25 \mathrm{mM}$ phosphate buffer 60:40 (v/v) mobile phase delivered at $1.5 \mathrm{~mL} / \mathrm{min}$, the observed retention time of $\left[{ }^{18} \mathrm{~F}\right]$ fluoride was between 2 and $3 \mathrm{~min}, 6 \mathrm{~min}$ for $\left[{ }^{18} \mathrm{~F}\right] \mathrm{FBB}$, and $14 \mathrm{~min}$ for the fluorinated intermediate.

\section{Droplet synthesis platform}

Radiochemistry was performed in droplet format using Teflon-coated silicon chips that had small circular regions of Teflon etched away, leaving hydrophilic patches that act as surfacetension traps to confine reagents during the multi-step radiosynthesis. Temperature control was achieved by affixing the chip atop a ceramic heater with thermal paste. The details of the chip fabrication were previously reported (Wang et al. 2017). Initially, the conditions were optimized manually using chips containing 4 reaction sites (Rios et al. 2019) on a platform with 4 heaters. Based on optimized conditions, the synthesis was adapted onto an ultra-compact automated droplet radiosynthesizer (Wang et al. 2019a) allowing for reduced radiation exposure and operation time via automation.

The overall setup comprises a droplet synthesizer, analytical-scale HPLC purification, and a newly-developed automated solid-phase extraction setup to perform formulation using custom micro-cartridges with C18 resin (Fig. 1). Details of the formulation system and cartridge fabrication are provided in the Supporting information, sections 1 and 2, respectively. Briefly, the inlet of the cartridge was connected to a selector valve and the outlet to a 3-way valve. Using the selector valve, different solutions could be flowed through the cartridge such as the $\left[{ }^{18} \mathrm{~F}\right] \mathrm{FBB}$ fraction vial (trapping step), a vial with aqueous sodium ascorbate solution (washing step), and a vial with ethanol (elution step). The 3-way valve was used to direct the cartridge output to waste (trapping and washing steps) or the product vial (elution step). The liquid movement was initiated by 


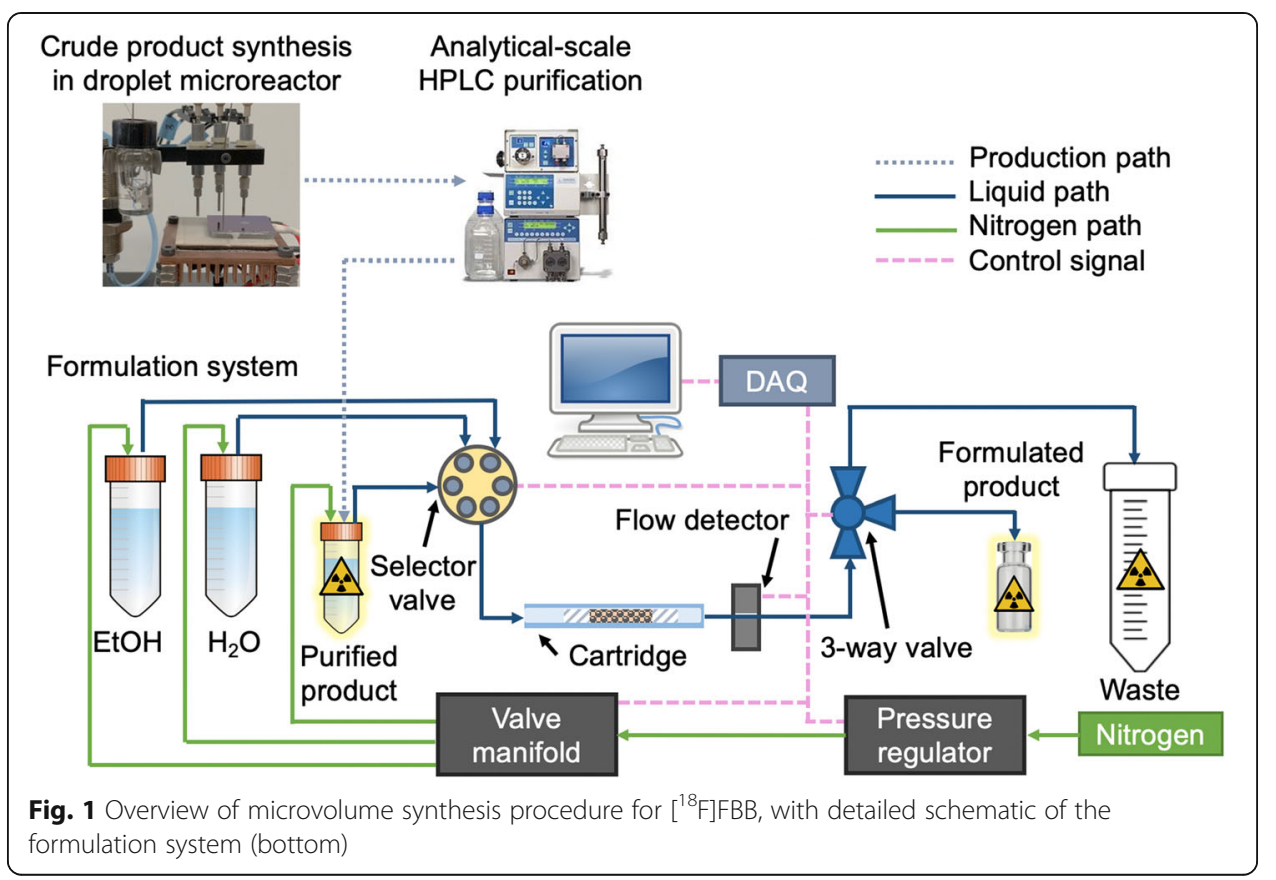

applying nitrogen pressure to the vials containing $\left[{ }^{18} \mathrm{~F}\right] \mathrm{FBB}(15 \mathrm{psi})$, water (15 psi) and ethanol (3 psi). A program written in LabView (National Instruments, Austin, TX) automatically controlled the valves and pressure sources via a data acquisition module (DAQ) to complete the trapping, washing, and elution steps.

\section{Microvolume radiosynthesis}

\section{Preliminary synthesis optimization}

The microvolume synthesis was adapted from the common 2-step approach, which consists of fluorination of the Boc-protected precursor using $\left[{ }^{18} \mathrm{~F}\right] \mathrm{KF} / \mathrm{K}_{222}$, followed by a hydrolysis step (Zhang et al. 2005). A schematic representation of the microvolume synthesis process is shown in Fig. 2.

Initial pre-optimization conditions for microvolume synthesis were selected by scaling down the conventional synthesis conditions reported in Collins et al. (Collins et al. 2017). The fluorination reaction volume was reduced 90 -fold from $1.8 \mathrm{~mL}$ to $20 \mu \mathrm{L}$, but the precursor concentration was maintained at $4 \mathrm{mM}$, resulting in a precursor amount of 120 nmol. The fluorination solvent was changed from $\mathrm{MeCN}$ to DMSO, since MeCN evaporated too quickly in droplet format. The total amount of cryptand phase-transfer catalyst was reduced 180-fold (from $49 \mu \mathrm{mol}$ to $275 \mathrm{nmol}$ of $\mathrm{K}_{2} \mathrm{CO}_{3}$ and from $68 \mu \mathrm{mol}$ to $383 \mathrm{nmol}$ of $\mathrm{K}_{222}$ ). First, the effect of temperature on the fluorination reaction was studied, followed by optimization of the amount of $\mathrm{K}_{2} \mathrm{CO}_{3} / \mathrm{K}_{222}$, and then amount of precursor. Each set of conditions was repeated $n=4$ times, with reagents delivered manually via pipette.

For each experiment, aqueous $\left[{ }^{18} \mathrm{~F}\right]$ fluoride $(10-20 \mu \mathrm{L} ; \sim 7.4-370 \mathrm{MBq})$ was mixed with the desired amount of $\mathrm{K}_{222} / \mathrm{K}_{2} \mathrm{CO}_{3}$ in $4.5 \mu \mathrm{L} \mathrm{H} \mathrm{H}_{2} \mathrm{O}$ and loaded to the reaction site to be evaporated to dryness at $100^{\circ} \mathrm{C}$ for $2 \mathrm{~min}$. Next the desired amount of precursor in DMSO was added to the dried fluoride residue and reacted at the desired temperature for $5 \mathrm{~min}$. For initial optimization experiments the crude product of the 


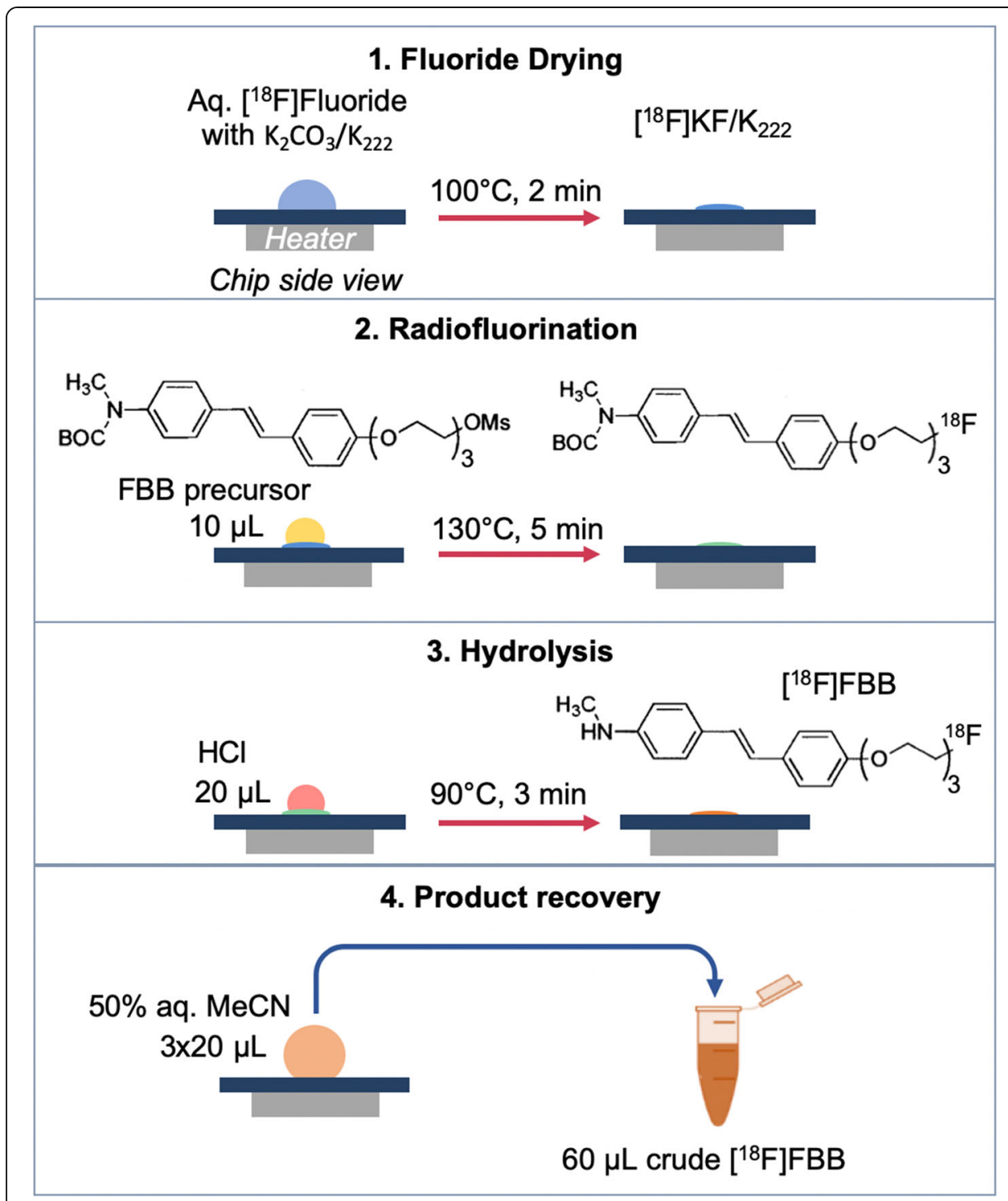

Fig. 2 Schematic of the microscale synthesis of crude $\left[{ }^{18} \mathrm{~F}\right] \mathrm{FBB}$

fluorination reaction was collected and analyzed. In other cases, the hydrolysis step was performed by adding $20 \mu \mathrm{L}$ of $1 \mathrm{~N} \mathrm{HCl}$ to the reaction mixture and heating at $90{ }^{\circ} \mathrm{C}$ for 3 mins. To recover the crude product (or intermediate), $20 \mu \mathrm{L}$ of collection mixture was added to resuspend the product on chip, and then transferred into the crude product vial. To ensure thorough recovery from the chip, the collection procedure was repeated 2 more times ( 3 more times for the automated setup). To avoid radiolysis and photodegradation, the crude product vial was preloaded with $64 \mu \mathrm{L}$ of water with 33 $\mathrm{mg} / \mathrm{mL}$ of dry scavenger mix and kept in the dark.

\section{Automated microvolume synthesis}

The automated synthesis of $\left[{ }^{18} \mathrm{~F}\right] \mathrm{FBB}$ was performed on identical chips, but using a custom-built platform (Wang et al. 2019a) that supported automated reagent 
dispensing and product recovery. The reaction conditions were identical to the optimized manual synthesis conditions, except that the deprotection was performed using $1 \mathrm{M} \mathrm{HCl}: M e C N ~ 1: 1(\mathrm{v} / \mathrm{v})$. This $20 \mu \mathrm{L}$ acidic mixture was dispensed at the beginning of deprotection and another $20 \mu \mathrm{L}$ after $1.5 \mathrm{~min}$. The diluted acid was used to reduce damage to the reagent dispensers.

\section{Purification and formulation}

To perform purification, the crude product collected in aqueous scavenger solution was diluted with aqueous sodium phosphate buffer to a total volume of $175 \mu \mathrm{L}$ and delivered into an analytical radio-HPLC system with $200 \mu \mathrm{L}$ injection loop, and separated as described in the "Analytical methods" section. The $\left[{ }^{18} \mathrm{~F}\right] \mathrm{FBB}$ peak (retention time 6 min) was collected (for 1.0-1.5 min) into a $50 \mathrm{~mL}$ conical tube (Falcon, Corning, USA) pre-loaded with $33 \mathrm{mg} / \mathrm{mL}$ dry scavenger mix in $3 \mathrm{~mL}$ water and covered by aluminum foil.

Formulation was performed by diluting the purified $\left[{ }^{18} \mathrm{~F}\right] \mathrm{FBB}$ with $30 \mathrm{~mL}$ of DI water, and carrying out solid phase extraction (SPE) using a miniature C18 cartridge made by packing $\mathrm{C} 18$ resin (5 or $10 \mathrm{mg}$ ) into lengths of tubing (Supporting Information, Section 1). Preconditioning of miniature cartridges was performed with $5 \mathrm{~mL} \mathrm{MeOH}$ followed by $6 \mathrm{~mL}$ of DI water at approximately $1 \mathrm{~mL} / \mathrm{min}$. An automated solid-phase extraction setup was built to perform the formulation step (Fig. 1). Details of this setup can be found in the Supporting Information, Section 2. In the final formulation procedure, the diluted $\left[{ }^{18} \mathrm{~F}\right] \mathrm{FBB}$ was trapped on the cartridge, and then the cartridge was washed by flowing through $10 \mathrm{~mL}$ DI water containing $10 \mathrm{mg} / \mathrm{mL}$ of dry scavenger mix to remove residual solvents and impurities. (The amount of scavenger is the same as reported by Rominger et al. (Rominger et al. 2013)). Finally, the trapped $\left[{ }^{18} \mathrm{~F}\right] \mathrm{FBB}$ was eluted from the cartridge using $150 \mu \mathrm{L}$ EtOH into an amber-colored glass product vial preloaded with $850 \mu \mathrm{L}$ of stabilization solution.

\section{Evaluation of synthesis performance}

Multiple measurements were collected during the synthesis to calculate several parameters related to the synthesis performance. Unless otherwise specified, all reported percentage values (yields, efficiencies) are decay-corrected (d.c.). Starting activity was determined by calculating a difference in activity measurements of a source vial before and after addition of the radionuclide from the vial to the chip (accounting for losses in pipette tips). Collection efficiency is the ratio of activity of the crude reaction mixture recovered from the chip relative to the starting activity. Residual chip activity is the percentage of starting activity that remained on the chip after the synthesis and crude reaction mixture recovery. Radiochemical conversion is the percentage of the desired product $\left(\left[{ }^{18} \mathrm{~F}\right] \mathrm{FBB}\right)$ in the crude mixture as determined by radio-TLC. Crude $\left[{ }^{18} \mathrm{~F}\right] \mathrm{FBB}$ radiochemical yield (crude RCY) is calculated by multiplying the collection efficiency by the radiochemical conversion. Isolated yield is the ratio of the activity of the purified product collected after HPLC purification to the starting activity. Formulated product yield is calculated by dividing the activity of the final formulated product by the starting activity. 
To carefully evaluate the formulation performance, additional parameters were calculated relative to the activity of pure $\left[{ }^{18} \mathrm{~F}\right] \mathrm{FBB}$ fraction obtained after HPLC purification. The formulation efficiency is the ratio of formulated product activity to the activity of the $\left[{ }^{18} \mathrm{~F}\right] \mathrm{FBB}$ fraction. Activity in waste is the ratio of activity in the waste container relative to the $\left[{ }^{18} \mathrm{~F}\right] \mathrm{FBB}$ fraction, and fraction collection vial residual activity is the percentage of activity remaining in the initial $\left[{ }^{18} \mathrm{~F}\right] \mathrm{FBB}$ fraction vial after the formulation process is complete. Cartridge residual activity is the percentage of the initial $\left[{ }^{18} \mathrm{~F}\right] \mathrm{FBB}$ activity that remained on the cartridge after formulation. Residual in the system is the percentage of the initial $\left[{ }^{18} \mathrm{~F}\right] \mathrm{FBB}$ fraction that was not recovered (i.e. remaining in various portions of the formulation system, e.g. valves, tubing, etc.).

\section{Results}

\section{Microvolume synthesis optimization}

The influence of fluorination temperature, base amount, and precursor concentration on the fluorination step (as measured by the resulting amount of fluorinated intermediate) is summarized in Fig. 3. Fluorination efficiency increased as temperature was increased from 90 to $130{ }^{\circ} \mathrm{C}$, and while there was a very slight reduction in collection efficiency over this temperature range, the overall $\mathrm{RCY}$ of the intermediate increased with increasing temperature (Fig. 3a). We thus chose $130^{\circ} \mathrm{C}$ as the optimal reaction temperature. The amount of base had a more complex influence on reaction performance (Fig. 3b). At low base amount $\left(<70 \mathrm{nmol} \mathrm{K}_{2} \mathrm{CO}_{3} / 100 \mathrm{nmol} \mathrm{K} 222\right)$, the collection efficiency, fluorination efficiency and RCY of the intermediate increased strongly with base amount, while at moderate amounts $\left(70-280 \mathrm{nmol} \mathrm{K}_{2} \mathrm{CO}_{3} / 100-390 \mathrm{nmol} \mathrm{K} 222\right)$, there was relatively little change and the RCY remained nearly constant. Higher amounts of base led to a gradual reduction in fluorination efficiency and RCY of the intermediate (though collection efficiency was constant). Based on this data, the optimal base quantity was $275 \mathrm{nmol} \mathrm{K}_{2} \mathrm{CO}_{3} / 383 \mathrm{nmol} \mathrm{K} 222$. For low precursor amount (< $40 \mathrm{nmol}$ ), there was a rapid increase in fluorination efficiency (and RCY of the intermediate) as precursor amount was increased, and at higher amounts, the slight decrease in collection efficiency canceled out the slight increase in fluorination efficiency

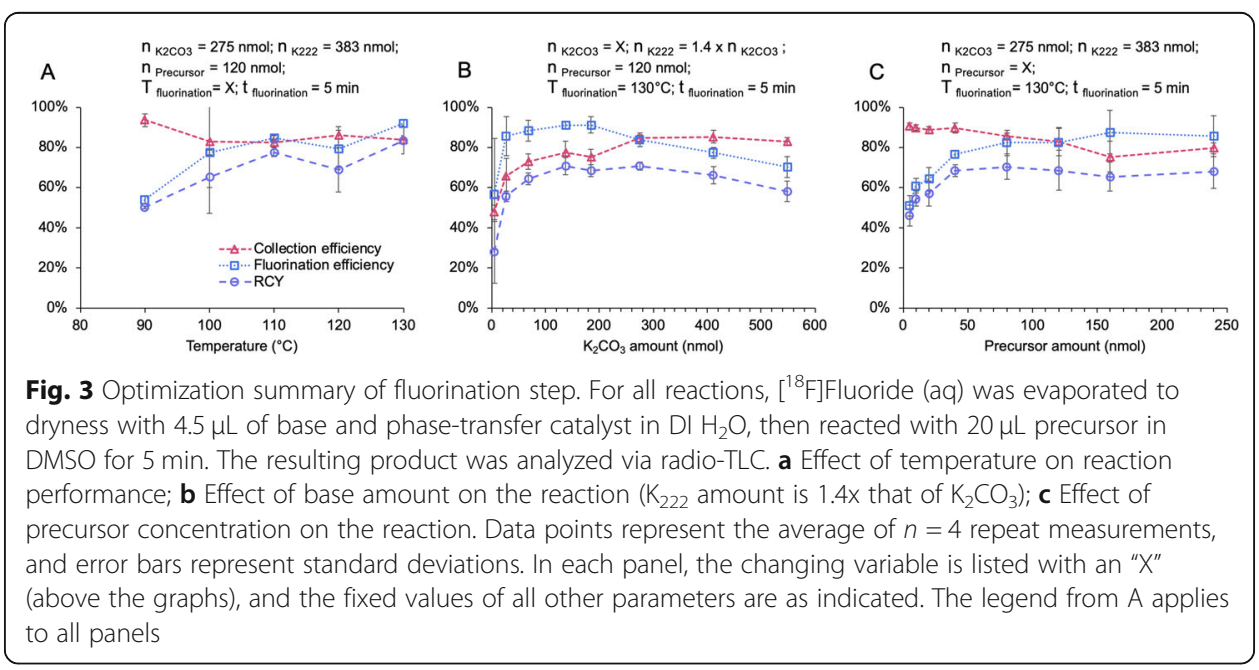


resulting in nearly constant RCY of the intermediate (Fig. 3c). We chose $80 \mathrm{nmol}$ of precursor as the optimum value. Using the optimal conditions together, the RCY of the fluorinated intermediate (without deprotection) was $70 \pm 6 \%(n=4)$.

It was observed that there was a residual volume of DMSO left at the end of the fluorination reaction. Concerned that this DMSO would adversely affect downstream purification on the analytical scale HPLC (as observed previously in our lab) and would dilute the deprotection solution, we opted later to reduce the reaction volume to $10 \mu \mathrm{L}$. Based on our optimization data seen in Fig. 3, we did not expect much change in performance by this effective doubling of base and precursor concentrations.

After this change, the amount of residual DMSO was reduced, and after deprotection with $20 \mu \mathrm{L}$ of $1 \mathrm{M} \mathrm{HCl}$, the crude $\mathrm{RCY}$ of $\left[{ }^{18} \mathrm{~F}\right] \mathrm{FBB}$ was $63 \pm 6 \%(n=4)$ (i.e., $66 \pm 6 \%$ collection efficiency and $96 \pm 1 \%$ radiochemical conversion). When the synthesis was automated and performed at similar activity levels $(\sim 7.4 \mathrm{MBq}$ starting activity), we observed a crude RCY of $58 \pm 7 \%(n=5)$ (i.e., $69 \pm 9 \%$ collection efficiency, $86 \pm 9 \%$ radiochemical conversion).

To produce quantities compatible with preclinical applications, further experiments were carried out with $\sim 300 \mathrm{MBq}$ starting activity level and the results are summarized in Table 1. Using the manual procedure for crude synthesis, the crude RCY was $78 \pm 6$ $(n=3)$ (i.e. $87 \pm 1 \%$ collection efficiency and $88 \pm 6 \%$ radiochemical conversion). The automated synthesis with $\sim 300 \mathrm{MBq}$ starting activity resulted in $60 \pm 5 \%(n=3)$ crude RCY (83 $\pm 3 \%$ collection efficiency, $72 \pm 4 \%$ radiochemical conversion).

\section{Purification and formulation Purification}

The crude $\left[{ }^{18} \mathrm{~F}\right] \mathrm{FBB}$ was purified using isocratic analytical scale radio-HPLC. The product was collected in $\sim 3 \mathrm{~mL}$ volume, taking $\sim 12 \mathrm{~min}$ for purification. In manual runs with $\sim 300 \mathrm{MBq}$ of starting activity, the isolated yield after purification was $61 \pm 2 \%$ $(n=3)$ with molar activity of $370 \pm 60 \mathrm{GBq} / \mu \mathrm{mol}$ decay-corrected to the end of purification (Table 1). Similarly, the equivalent experiments using automated procedure of the crude synthesis resulted in $53 \pm 8 \%(n=3)$ isolated yield and $260 \pm 80 \mathrm{GBq} / \mu \mathrm{mol}$ molar activity.

Table 1 Summary of synthesis performance when performed manually or on the automated setup. All \% values are calculated in reference to starting activity.

\begin{tabular}{|c|c|c|}
\hline & $\begin{array}{l}\text { Manual Synthesis } \\
n=3\end{array}$ & $\begin{array}{l}\text { Automated Synthesis } \\
n=3\end{array}$ \\
\hline Starting activity (MBq) & $260 \pm 100$ & $330 \pm 120$ \\
\hline Collection efficiency (\%) & $87 \pm 1$ & $83 \pm 3$ \\
\hline Residual chip activity (\%) & $0.4 \pm 0.2$ & $1.2 \pm 0.3$ \\
\hline Radiochemical conversion of the crude (\%) & $88 \pm 6$ & $72 \pm 4$ \\
\hline Crude $\left[{ }^{18} \mathrm{~F}\right] \mathrm{FBB}$ yield $(\%)$ & $78 \pm 6$ & $60 \pm 5$ \\
\hline Crude synthesis time (min) & $30 \pm 8$ & $28 \pm 1$ \\
\hline Isolated $\left[{ }^{18} \mathrm{~F}\right] \mathrm{FBB}$ yield (\%) & $61 \pm 2$ & $53 \pm 8$ \\
\hline Molar activity d.c. (GBq/ $/$ mol) & $370 \pm 60$ & $260 \pm 80$ \\
\hline
\end{tabular}

${ }^{a}$ Molar activity is reported at the end of HPLC purification 


\section{Formulation}

To ensure sufficient activity concentration of the formulated $\left[{ }^{18} \mathrm{~F}\right] \mathrm{FBB}$, we developed custom-made miniature C18 cartridges with small bed volumes that could be eluted with a significantly reduced amount of EtOH compared to conventional C18 Sep-pak cartridges. To assess trapping capacity during preliminary runs of the formulation protocol, we used $5 \mathrm{nmol}$ of FBB reference standard spiked with small amount of purified $\left[{ }^{18} \mathrm{~F}\right] \mathrm{FBB}(15 \mathrm{MBq})$ to simulate moderate activity levels. (In syntheses starting with $\sim 370 \mathrm{MBq}$ activity, we determined from the HPLC purification chromatogram that the mass of FBB was $0.7 \pm 0.3 \mathrm{nmol}(n=3)$, therefore addition of $5 \mathrm{nmol}$ would roughly correspond to $\mathrm{a} \sim 2.6 \mathrm{GBq}$ batch of $\left[{ }^{18} \mathrm{~F}\right] \mathrm{FBB}$.) When performing trapping with $5 \mathrm{mg}$ resin only $77 \%$ of the product activity was trapped on the cartridge and $19 \%$ of the activity was found in waste (untrapped), and $77 \%$ of the initial $\left[{ }^{18} \mathrm{~F}\right] \mathrm{FBB}$ was recovered at the end of elution. However, trapping was more efficient for the $10 \mathrm{mg}$ resin cartridge with only $1 \pm 1 \%(n=3)$ lost in waste (untrapped), and the elution recovered $91 \pm 5 \%(n=3)$ of the initial $\left[{ }^{18} \mathrm{~F}\right] \mathrm{FBB}$. Results are summarized in Table 2 . Due to the nearly quantitative trapping performance, $10 \mathrm{mg}$ cartridges were used for the remaining experiments.

These experiments were repeated using the automated formulation system (with 10 mg cartridge), resulting in $81 \pm 5 \%(n=3)$ recovery of the initial pure $\left[{ }^{18} \mathrm{~F}\right] \mathrm{FBB}$ using $150 \mu \mathrm{L} \mathrm{EtOH}$, which was collected into $850 \mu \mathrm{L}$ of stabilization solution. After 25 min formulation of $110 \pm 50 \mathrm{MBq}(n=3)$ of activity, there was $1 \mathrm{~mL}$ of $77 \pm 35 \mathrm{MBq}(n=3)$ of injection ready product. Minor activity losses were measured $3 \pm 3 \%(n=3)$ in waste after trapping and washing steps, and only $1 \pm 1 \%$ remained on the cartridge, however it was observed that $14 \pm 4 \%$ of the initial activity remained unaccounted for within the formulation system components (Table 2). Residual activity in the formulation system components was difficult to accurately measure, but substantial portions were found in the fluid path used in trapping. In the future it can be possible to perform additional rinsing step to improve product recovery.

In summary, after total of 55 min synthesis time (30 for crude synthesis, 8 min for purification and $17 \mathrm{~min}$ for formulation) the radiochemically pure (>98\%) $\left[{ }^{18} \mathrm{~F}\right] \mathrm{FBB}$ was

Table 2 Summary of performance of the formulation step. All \% values are calculated in reference to starting pure $\left[{ }^{18} \mathrm{~F}\right] \mathrm{FBB}$ activity

\begin{tabular}{llll}
\hline & $\begin{array}{l}\text { Manual } \\
\text { Formulation }^{\text {a }}\end{array}$ & $\begin{array}{l}\text { Manual } \\
\text { Formulation }^{\text {a }}\end{array}$ & $\begin{array}{l}\text { Automated } \\
\text { Formulation }\end{array}$ \\
\hline Mass of C18 resin in cartridge (mg) & 5 & 10 & 10 \\
Number of repeats & $n=1$ & $n=3$ & $n=3$ \\
Starting pure $\left[{ }^{18} \mathrm{~F}\right] \mathrm{FBB}$ activity (MBq) & 7.4 & $15 \pm 11$ & $112 \pm 51$ \\
Formulation efficiency (\%) & 77 & $91 \pm 5$ & $81 \pm 5$ \\
Formulation time (min) & 18 & 22 & 17 \\
Losses: & & & $3 \pm 3$ \\
Activity in waste (\%) & 19 & $1 \pm 1$ & $2 \pm 1$ \\
Fraction collection vial residual activity (\%) & 3 & $6 \pm 4$ & $1 \pm 1$ \\
Cartridge residual activity (\%) & 0 & $0 \pm 1$ & $14 \pm 4$ \\
Residual in the system (\%) & N/A & N/A & \\
\hline
\end{tabular}

${ }^{\mathrm{a}}$ Initial manual formulation runs were performed using $5 \mathrm{nmol}$ of $\mathrm{FBB}$ spiked with purified $\left[{ }^{18} \mathrm{~F}\right] \mathrm{FBB}$ product, to mimic the mass loading of a higher activity level, while working with less radioactivity 
acquired in $49 \pm 3 \%(n=3)$ yield with measured $340 \pm 60 \mathrm{GBq} / \mu \mathrm{mol}$ molar activity at the end of synthesis.

\section{Discussion}

This work shows that the synthesis of $\left[{ }^{18} \mathrm{~F}\right] \mathrm{FBB}$ can be performed in a microvolume reactor in relatively short time and with excellent yield and molar activity.

\section{High-throughput optimization}

Initial optimization of the reaction was performed using high-throughput techniques (i.e., multiple reactions in parallel) (Rios et al. 2019), allowing the study of multiple reaction parameters with a high number of replicates. Notably, testing 21 sets of conditions (altering 3 parameters) with $n=4$ repeats for each condition (a total of 84 experiments) was performed in only 6 radiochemistry sessions $(12 \mathrm{~h})$. Optimization of each parameter was performed sequentially in a one variable at a time (OVAT) fashion, and it is possible that additional iterations of optimization using parameters from the previous round, or more efficient and comprehensive optimization approaches such as design of experiments (DoE), could yield slight improvements, but this was not explored here.

Having extensive data about the overall impact of each parameter can be used to increase the robustness of a synthesis, i.e. by choosing parameter values where the performance is insensitive to variances in the variable (close to horizontal slope). While our optimization focused on yield, the data could also be used to optimize other outcomes, for example, minimizing the amount of precursor (to minimize cost or simplify purification) while achieving an acceptable yield.

\section{Purification and formulation}

In this work purification was performed via analytical scale HPLC, which has been previously reported for purification of tracers produced in microfluidic systems (Lebedev et al. 2012; Wang et al. 2017; Javed et al. 2014; Wang et al. 2019a; Lisova et al. 2019; Wang et al. 2019b). Analytical-scale purification allows very short purification times (several min) and small volume $(\sim 1-2 \mathrm{~mL})$ of pure fraction. The simplest purification and formulation route for $\left[{ }^{18} \mathrm{~F}\right]$ Florbetaben (and the one recommended for the Life Molecular Imaging kits) would be purification via ethanolic mobile phase (sodium ascorbate buffer:EtOH, 40:60, v/v) such that the collected fraction can be formulated simply by dilution. However, when scaling to an analytical HPLC system, the pressure limit was exceeded for amounts of $\mathrm{EtOH}>20 \%(\mathrm{v} / \mathrm{v})$ at $1 \mathrm{~mL} / \mathrm{min}$, and the late retention time of $\left[{ }^{18} \mathrm{~F}\right] \mathrm{FBB}$ when using lower amounts of $\mathrm{EtOH}$ was not practical. It is possible that other column types or sizes could avoid this problem, but we instead used an MeCN-based mobile phase for separation, which required a downstream formulation step to remove the acetonitrile after purification. Under this condition, the crude product was rapidly and efficiently purified and collected within $8 \mathrm{~min}$ of injecting the crude reaction mixture.

Initially, we tried evaporative solvent exchange to remove $\mathrm{MeCN}$, performing evaporation at $100{ }^{\circ} \mathrm{C}$ followed by resuspension of the dried product in PBS. However, evaporation of $2-3 \mathrm{~mL}$ of volume of collected crude product took a significant amount of 
time (15 min). We also observed the solution change from clear to cloudy, and after resuspension in formulation buffer, a significant amount of product $(\sim 70 \%$ of initial pure $\left.\left[{ }^{18} \mathrm{~F}\right] \mathrm{FBB}\right)$ was stuck to the vial, likely due to the poor solubility of this compound.

Instead, formulation via solid-phase extraction was explored using a C18 cartridge as widely reported by others (Table 3) (Rominger et al. 2013; Patt et al. 2010; Rowe et al. 2008). However, commercial C18 cartridges (Waters Sep-pak C18 Plus Light, 130 mg, Waters Corporation, Milford, MA) were not suitable for small batch syntheses: to efficiently elute the product from the cartridge, 1-2 $\mathrm{mL} \mathrm{EtOH}$ was needed, which would then be diluted with stabilizing solution to a total volume of $7-14 \mathrm{~mL}$ to lower the $\mathrm{EtOH}$ concentration to $15 \%$ (acceptable limit for the formulation of this probe). For preparation of small batches of $\left[{ }^{18} \mathrm{~F}\right] \mathrm{FBB}$ (e.g. $<185 \mathrm{MBq}$ ), the resulting concentration $(<26 \mathrm{MBq} / \mathrm{mL})$ would be too dilute for small animal imaging. Instead, we explored the use of custom miniature cartridges made by packing C18 resin into lengths of tubing (Supporting Information, Section 1), which allowed minimization of the final formulated volume. Cartridges made inside 0.02 " ID tubing exhibited extremely low flow rates, but cartridges packed inside 0.0625 " ID tubing had suitable flow rates. With these miniature cartridges, the product could be efficiently recovered with only $150 \mu \mathrm{L}$ $\mathrm{EtOH}$, enabling formulation (to dilute $\mathrm{EtOH}$ to an acceptable level of 15\%) in a final volume of $1 \mathrm{~mL}$.

Unfortunately, the formulation requires extra time $(17 \mathrm{~min})$, leading to some radioactive decay during the process. The formulation process was very efficient when performed manually, however the presence of extra fluidic components in the automated system resulted in slightly reduced efficiency. Additional losses in the automated formulation system resulted in a 10 point drop in formulation efficiency compared to manual method (i.e. $81 \%$ versus $91 \%$ ).

In the current formulation protocol, the trapping step takes up the majority of time, requiring $\sim 10 \mathrm{~min}$ in total to process nearly $30 \mathrm{~mL}$ of volume, while the washing and elution steps take 3 and $4 \mathrm{~min}$, respectively. Possible directions for improvement would be to optimize the HPLC purification method to reduce peak width and collection volume, leading to a lower dilution volume, or optimizing the applied pressure during trapping to achieve both highest trapping efficiency and flow rate.

\section{Probe stability and radiolysis}

It has been reported that $\mathrm{N}$-methylaniline substituents can make this, and structurally similar, beta-amyloid PET tracers, quite susceptible to radiolysis (Scott et al. 2009). In fact, significant radiolysis (8 point reduction in radiochemical purity) was already observed within $10 \mathrm{~min}$ for $\left[{ }^{18} \mathrm{~F}\right] \mathrm{AV}-19$ in isotonic saline with $5 \% \mathrm{EtOH}$ at a modest activity concentration of $185 \mathrm{MBq} / \mathrm{mL}$. Scott et al. found that addition of appropriate radical scavengers extended stability of the compound to multiple hours even in larger scale productions yielding 7.4 GBq of product (Scott et al. 2009).

To ensure highest radiochemical purity and stability of $\left[{ }^{18} \mathrm{~F}\right] \mathrm{FBB}$ produced with microvolume method, the scavenger had to be introduced at various steps of the production. Using literature reports as a guide (Rominger et al. 2013; Scott et al. 2009), scavenger was introduced into the collection solution during collection of the crude product from the chip, pre-loaded in the collection vial prior to recovery of the pure 


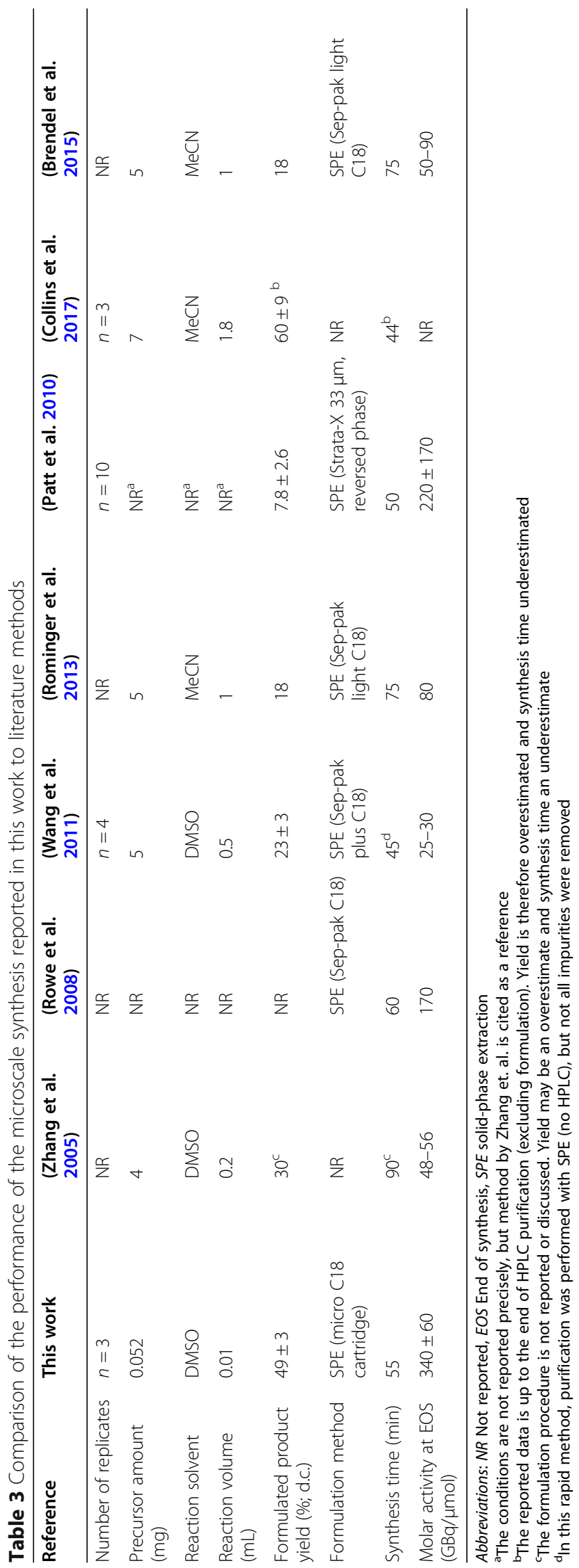


fraction from HPLC, in the dilution solution used prior to formulation, and pre-loaded in the final product vial.

\section{Benefits of microscale synthesis}

The whole production (synthesis, purification, formulation) can be completed in 55 min with high yield ( $50 \%$ decay-corrected). The 2-step reaction itself was quite fast, due largely to the small volume reaction which requires very little time for heating/cooling or performing solvent evaporation steps (e.g. during $\left[{ }^{18} \mathrm{~F}\right]$ fluoride drying). Though not explored here, there is room to improve and develop a faster analytical-scale HPLC separation process, and to shorten the formulation process as discussed in detail above. It is also feasible to increase the amount of starting activity (i.e. loading larger volume of $\left[{ }^{18} \mathrm{~F}\right]$ fluoride or using a radioisotope concentrator (Chao et al. 2018)) to produce higher amounts of the probe.

Another advantage of the microscale synthesis is that high molar activity 340 $\mathrm{GBq} / \mu \mathrm{mol}$ could be achieved at the end of synthesis, even when starting with low amounts $(<350 \mathrm{MBq})$ of $\left[{ }^{18} \mathrm{~F}\right]$ fluoride, something that cannot be achieved with conventional macroscale synthesis processes (Sergeev et al. 2018). Due to the importance of high molar activity when imaging certain tracers in small animals, the ability to achieve high molar activity in small batches can be a huge advantage, avoiding significant waste of radionuclide and probe that would otherwise be needed (i.e. to prepare a large batch to ensure high molar activity, but discard most of it since only a small quantity is needed for animal imaging). High molar activity of the amyloid tracer $\left[{ }^{11} \mathrm{C}\right] \mathrm{PIB}$ has been reported to be particularly important for detection of relatively immature amyloid lesions in small animal models (Maeda et al. 2007), suggesting it could be critical in certain cases for $\left[{ }^{18} \mathrm{~F}\right] \mathrm{FBB}$ imaging as well. Additionally, high molar activity provides a longer duration over which the molar activity can be adjusted to be within a desired range for experiments, and helps to lower the total injected mass of the tracer.

In comparison with literature methods (Table 3), the yield of this microvolume synthesis is among the highest, while only consuming $\sim 1 \%$ the amount of precursor as other methods. In combination with the simple apparatus and compact size, this could lead to lower costs in production of $\left[{ }^{18} \mathrm{~F}\right] \mathrm{FBB}$, and potentially enable new models of production and distribution for preclinical research. The microvolume synthesis approach could also provide a way for existing production facilities to add capability for production of additional tracers without significant need for additional hot cell space.

\section{Conclusion}

We implemented an efficient synthesis of $\left[{ }^{18} \mathrm{~F}\right] \mathrm{FBB}$ in microdroplet format on simple chips with surface-tension traps acting as reaction sites. The overall synthesis was fast (55 min), high-yielding $(49 \pm 3 \%, n=3)$, and had high molar activity $(340 \pm 60 \mathrm{GBq} /$ $\mu \mathrm{mol}, n=3)$. The combination of a droplet radiosynthesizer with analytical-scale radioHPLC purification system, and a miniature SPE-based formulation system, provides a platform for streamlined and economical production of $\left[{ }^{18} \mathrm{~F}\right] \mathrm{FBB}$, and could be extended to other tracers in a straightforward manner. 


\section{Supplementary Information}

Supplementary Information accompanies this paper at https://doi.org/10.1186/s41181-020-00113-w.

Additional file 1.

\section{Abbreviations}

PET: Positron emission tomography; SPE: Solid phase extraction; TLC: Thin-layer chromatography; HPLC: High performance liquid chromatography; DI: Deionized; RCY: Radiochemical yield; D.c.: Decay-corrected; ID: Inner diameter; DAQ: Data acquisition

\section{Acknowledgements}

We thank Roger Slavik and the staff of the UCLA Biomedical Cyclotron Facility for generously providing $\left[{ }^{18} \mathrm{~F}\right]$ fluoride for these studies. We thank Sofie, Inc. and Life Molecular Imaging GmbH for generously supplying the synthesis kits and precursor for these studies. Microfluidic substrates were produced in the UCLA Integrated NanoSystems Cleanroom (ISNC), and we thank the staff for technical support.

\section{Authors' contributions}

$\mathrm{KL}$ performed optimization of the tracer production and analyzed data. KL and JW performed radiochemistry. $\mathrm{KL}$ and PHC designed and built micro-cartridge device. KL and RMVD designed experiments. RMVD oversaw the research project. All authors contributed to writing and revising the manuscript, and all authors approved the final version of the manuscript.

\section{Funding}

This work was supported in part by the National Cancer Institute (R21 CA212718, R33 CA240201), the National Institute on Aging (R21 AG049918), the National Institute of Mental Health (R44 MH 097271), the National Institute of Biomedical Imaging and Bioengineering (T32 EB002101), and the UCLA Foundation from a donation made by Ralph and Marjorie Crump for the Crump Institute for Molecular Imaging.

\section{Availability of data and materials}

Most of the experimental data is reported in the summary tables in the manuscript, and representative analytical chromatograms are shown in the Supplemental information. The additional datasets for each individual experiment are available from the corresponding author on reasonable request.

\section{Ethics approval and consent to participate}

This article does not contain any studies with human participants or animals performed by any of the authors.

\section{Consent for publication}

Not applicable.

\section{Competing interests}

Dr. van Dam is a founder of Sofie, Inc. The Regents of the University of California have licensed technology to Sofie related to this work that was invented by Dr. van Dam, and have taken equity in Sofie as part of the licensing transaction.

\section{Author details}

${ }^{1}$ Physics \& Biology in Medicine Interdepartmental Graduate Program, University of California Los Angeles, Los Angeles, CA, USA. ${ }^{2}$ Crump Institute for Molecular Imaging, University of California Los Angeles, Los Angeles, CA, USA.

${ }^{3}$ Department of Molecular \& Medical Pharmacology, University of California Los Angeles, Los Angeles, CA, USA.

${ }^{4}$ Department of Bioengineering, University of California Los Angeles, Los Angeles, CA, USA.

Received: 20 September 2020 Accepted: 20 November 2020

Published online: 04 December 2020

\section{References}

Blume T, Focke C, Peters F, Deussing M, Albert NL, Lindner S, et al. Microglial response to increasing amyloid load saturates with aging: a longitudinal dual tracer in vivo $\mu$ PET-study. J Neuroinflammation. 2018;15:307.

Brendel M, Jaworska A, Grießinger E, Rötzer C, Burgold S, Gildehaus F-J, et al. Cross-sectional comparison of small animal [18F]-Florbetaben amyloid-PET between transgenic AD mouse models. PLoS One. 2015;10:e0116678.

Brendel M, Jaworska A, Overhoff F, Blume T, Probst F, Gildehaus F-J, et al. Efficacy of chronic BACE1 inhibition in PS2APP mice depends on the regional A $\beta$ deposition rate and plaque burden at treatment initiation. Theranostics. 2018;8:4957-68

Brendel M, Probst F, Jaworska A, Overhoff F, Korzhova V, Albert NL, et al. Glial Activation and Glucose Metabolism in a Transgenic Amyloid Mouse Model: A Triple-Tracer PET Study. J Nucl Med. 2016;57:954-60.

Chao PH, Lazari M, Hanet S, Narayanam MK, Murphy JM, van Dam RM. Automated concentration of [18F]fluoride into microliter volumes. Appl Radiat Isot. 2018;141:138-48.

Collins J, Waldmann CM, Drake C, Slavik R, Ha NS, Sergeev M, et al. Production of diverse PET probes with limited resources: $24{ }^{18} \mathrm{~F}$-labeled compounds prepared with a single radiosynthesizer. PNAS. 2017;114:11309-14.

Dooraghi AA, Keng PY, Chen S, Javed MR, Kim C-J, AF CJC, et al. Optimization of microfluidic PET tracer synthesis with Cerenkov imaging. Analyst. 2013;138:5654-64. 
Elizarov AM, van Dam RM, Shin YS, Kolb HC, Padgett HC, Stout D, et al. Design and optimization of coin-shaped microreactor chips for PET radiopharmaceutical synthesis. J Nucl Med. 2010;51:282-7.

Fiel SA, Yang H, Schaffer P, Weng S, Inkster JAH, Wong MCK, et al. Magnetic droplet microfluidics as a platform for the concentration of [18F]fluoride and Radiosynthesis of Sulfonyl [18F]fluoride. ACS Appl Mater Interfaces. 2015;7:12923-9.

Hardy J, Selkoe DJ. The amyloid hypothesis of Alzheimer's disease: progress and problems on the road to therapeutics. Science. 2002;297:353-6.

Jagoda EM, Vaquero JJ, Seidel J, Green MV, Eckelman WC. Experiment assessment of mass effects in the rat: implications for small animal PET imaging. Nucl Med Biol. 2004;31:771-9.

Javed MR, Chen S, Lei J, Collins J, Sergeev M, Kim H-K, et al. High yield and high specific activity synthesis of [18F]fallypride in a batch microfluidic reactor for micro-PET imaging. Chem Commun. 2014;50:1192-4.

Keng PY, Chen S, Ding H, Sadeghi S, Shah GJ, Dooraghi A, et al. Micro-chemical synthesis of molecular probes on an electronic microfluidic device. PNAS National Acad Sci. 2012;109:690-5.

Keng PY, Sergeev M, van Dam RM. Advantages of Radiochemistry in Microliter Volumes. In: Kuge Y, Shiga T, Tamaki N, editors. Perspectives on Nuclear Medicine for Molecular Diagnosis and Integrated Therapy. Japan: Springer; 2016. [cited 2016 Apr 11]. p. 93-111. Available from: http://link.springer.com/chapter/10.1007/978-4-431-55894-1_7.

Kim H-K, Rashed Javed M, Chen S, A Zettlitz K, Collins J, M. Wu A, et al. On-demand radiosynthesis of N -succinimidyl-4-[ 18 F]fluorobenzoate ([ $18 \mathrm{~F}] \mathrm{SFB}$ ) on an electrowetting-on-dielectric microfluidic chip for $18 \mathrm{~F}$-labeling of protein. RSC Adv. 2019;9:32175-83.

Klunk WE, Engler H, Nordberg A, Wang Y, Blomqvist G, Holt DP, et al. Imaging brain amyloid in Alzheimer's disease with Pittsburgh compound-B. Ann Neurol. 2004;55:306-19.

Kung M-P, Kung HF. Mass effect of injected dose in small rodent imaging by SPECT and PET. Nucl Med Biol. 2005;32:673-8.

Lebedev A, Miraghaie R, Kotta K, Ball CE, Zhang J, Buchsbaum MS, et al. Batch-reactor microfluidic device: first human use of a microfluidically produced PET radiotracer. Lab Chip. 2012;13:136-45.

Lisova K, Chen BY, Wang J, Fong KM-M, Clark PM, van Dam RM. Rapid, efficient, and economical synthesis of PET tracers in a droplet microreactor: application to O-(2-[18F]fluoroethyl)-L-tyrosine ([18F]FET). EJNMMI radiopharm chem. 2019;5:1.

Lisova K, Sergeev M, Evans-Axelsson S, Stuparu AD, Beykan S, Collins J, et al. Microscale radiosynthesis, preclinical imaging and dosimetry study of [18FAMBF3-TATE: a potential PET tracer for clinical imaging of somatostatin receptors. Nucl Med Biol. 2018;61:36-44.

Maeda J, Ji B, Irie T, Tomiyama T, Maruyama M, Okauchi T, et al. Longitudinal, quantitative assessment of amyloid, Neuroinflammation, and anti-amyloid treatment in a living mouse model of Alzheimer's disease enabled by positron emission tomography. J Neurosci Society for Neuroscience. 2007;27:10957-68.

Ossenkoppele R, Jansen WJ, Rabinovici GD, Knol DL, van der Flier WM, van Berckel BNM, et al. Prevalence of amyloid PET positivity in dementia syndromes: a meta-analysis. JAMA. 2015;313:1939-50.

Patt M, Schildan A, Barthel H, Becker G, Schultze-Mosgau MH, Rohde B, et al. Metabolite analysis of [18F]Florbetaben (BAY 949172) in human subjects: a substudy within a proof of mechanism clinical trial. J Radioanal Nucl Chem. 2010;284:557-62.

Rios A, Wang J, Chao PH, van Dam RM. A novel multi-reaction microdroplet platform for rapid radiochemistry optimization. RSC Adv. 2019;9:20370-4.

Rominger A, Brendel M, Burgold S, Keppler K, Baumann K, Xiong G, et al. Longitudinal assessment of cerebral $\beta$-amyloid deposition in mice overexpressing Swedish mutant $\beta$-amyloid precursor protein using 18F-Florbetaben PET. J Nucl Med. 2013;54:1127-34.

Rowe CC, Ackerman U, Browne W, Mulligan R, Pike KL, O'Keefe G, et al. Imaging of amyloid $\beta$ in Alzheimer's disease with 18F-BAY94-9172, a novel PET tracer: proof of mechanism. Lancet Neurol. 2008;7:129-35.

Sabri O, Seibyl J, Rowe C, Barthel H. Beta-amyloid imaging with florbetaben. Clin Transl Imaging. 2015;3:13-26.

Sacher C, Blume T, Beyer L, Peters F, Eckenweber F, Sgobio C, et al. Longitudinal PET Monitoring of Amyloidosis and Microglial Activation in a Second-Generation Amyloid- $\beta$ Mouse Model. J Nucl Med. 2019;60:1787-93.

Scott PJH, Hockley BG, Kung HF, Manchanda R, Zhang W, Kilbourn MR. Studies into Radiolytic decomposition of Fluorine-18 labeled radiopharmaceuticals for positron emission tomography. Appl Radiat Isot. 2009;67:88-94.

Sergeev M, Lazari M, Morgia F, Collins J, Javed MR, Sergeeva O, et al. Performing radiosynthesis in microvolumes to maximize molar activity of tracers for positron emission tomography. Communications Chem. 2018;1:10.

Snellman A, Rokka J, López-Picón FR, Eskola O, Salmona M, Forloni G, et al. In vivo PET imaging of beta-amyloid deposition in mouse models of Alzheimer's disease with a high specific activity PET imaging agent [18F]flutemetamol. EJNMMI Res. 2014;4:37.

Wang H, Guo X, Jiang S, Tang G. Automated synthesis of [18F]Florbetaben as Alzheimer's disease imaging agent based on a synthesis module system. Appl Radiat Isot. 2013;71:41-6.

Wang H, Shi H, Yu H, Jiang S, Tang G. Facile and rapid one-step radiosynthesis of [18F]BAY94-9172 with a new precursor. Nucl Med Biol. 2011;38:121-7.

Wang J, Chao PH, Hanet S, van Dam RM. Performing multi-step chemical reactions in microliter-sized droplets by leveraging a simple passive transport mechanism. Lab Chip. 2017;17:4342-55.

Wang J, Chao PH, van Dam RM. Ultra-compact, automated microdroplet radiosynthesizer. Lab Chip. 2019a:2415-24.

Wang J, Holloway T, Lisova K, RM van D. Green and efficient synthesis of the radiopharmaceutical [18F]FDOPA using a microdroplet reactor. React Chem Eng. 2019b; [cited 2020 Jan 27]; Available from: https://pubs.rsc.org/en/content/ articlelanding/2020/re/c9re00354a.

Wang J, Rios A, Lisova K, Slavik R, Chatziioannou AF, van Dam RM. High-throughput radio-TLC analysis. Nucl Med Biol. 2020;82-83:41-8.

Yeo JM, Waddell B, Khan Z, Pal S. A systematic review and meta-analysis of 18F-labeled amyloid imaging in Alzheimer's disease. Alzheimers Dement (Amst). 2015;1:5-13.

Zhang W, Oya S, Kung M-P, Hou C, Maier DL, Kung HF. F-18 Polyethyleneglycol stilbenes as PET imaging agents targeting A aggregates in the brain. Nucl Med Biol. 2005;32:799-809.

\section{Publisher's Note}

Springer Nature remains neutral with regard to jurisdictional claims in published maps and institutional affiliations. 